\title{
Segmentation patterns in root apices
}

\author{
F. A. L. CLOWES
}

In an account of cell patterns in the root apex of Cyperus, $\mathrm{K}$ a d e $\mathrm{j}$ (1963) has drawn attention to an apparent dilemma in the interpretation of cell patterns that is of general interest. He shows that the root is subdivided into anticlinal segments which are seen most clearly in the cortex. He points out that pteridophyte roots with single apical cells have a similar segmentation and draws the conclusion that since this type of segmentation is derived from a single cell in pteridophytes the cortical segments in Cyperus must also be derived from a single cell. But Cyperus roots are large and presumably have a quiescent centre in the region where this single mother cell lies. If this cell is quiescent how does the segmentation arise?

All roots in which the cortex or cortex-epidermis complex is reduced to a single cell on the axis of the apex have this kind of segmentation (Fig. 1). The reason why the adventitious roots of Cyperus display segmentation in the cortex better than most others that have a similar construction, is that they are larger with a much greater widening of the cortex. This widening is so great that it causes the epidermis-cap boundary to become concave outwards on the axis of the root. In the space from the axis to a point twelve epidermal cells away the cortex-epidermis complex in one of Kadej's roots has expanded from one cell to 25 cells in width. At a similar number of epidermal cells from the axis in Zea (Fig. 1) the cortex-epidermis complex is only about 10 cells wide. The much greater importance of periclinal divisions in Cyperus emphasises the anticlinal segmentation of the cortex and so the pattern that catches the eye is different in the two species. Nevertheless anticlinal segmentation is present in all roots of this type though it is not usually so obvious as it is in Cyperus. Also, as $\mathrm{K}$ a d e $\mathrm{j}$ points out, the segmentation pattern occurs in the stele as well as the cortex. Again this is not so obvious as it is in the cortex, even in Cyperus, because the stele does not expand in width so much.

$\mathrm{K}$ a de j's conclusion that the segmentation of the cortex is due to this tissue's being derived from a single cell or a small group of cells is correct. The pattern of cells shows this clearly and there is no alternative explanation of the pattern. Secondly there is no doubt that $C y$ perus roots have a quiescent centre. The pattern of cells in these roots 


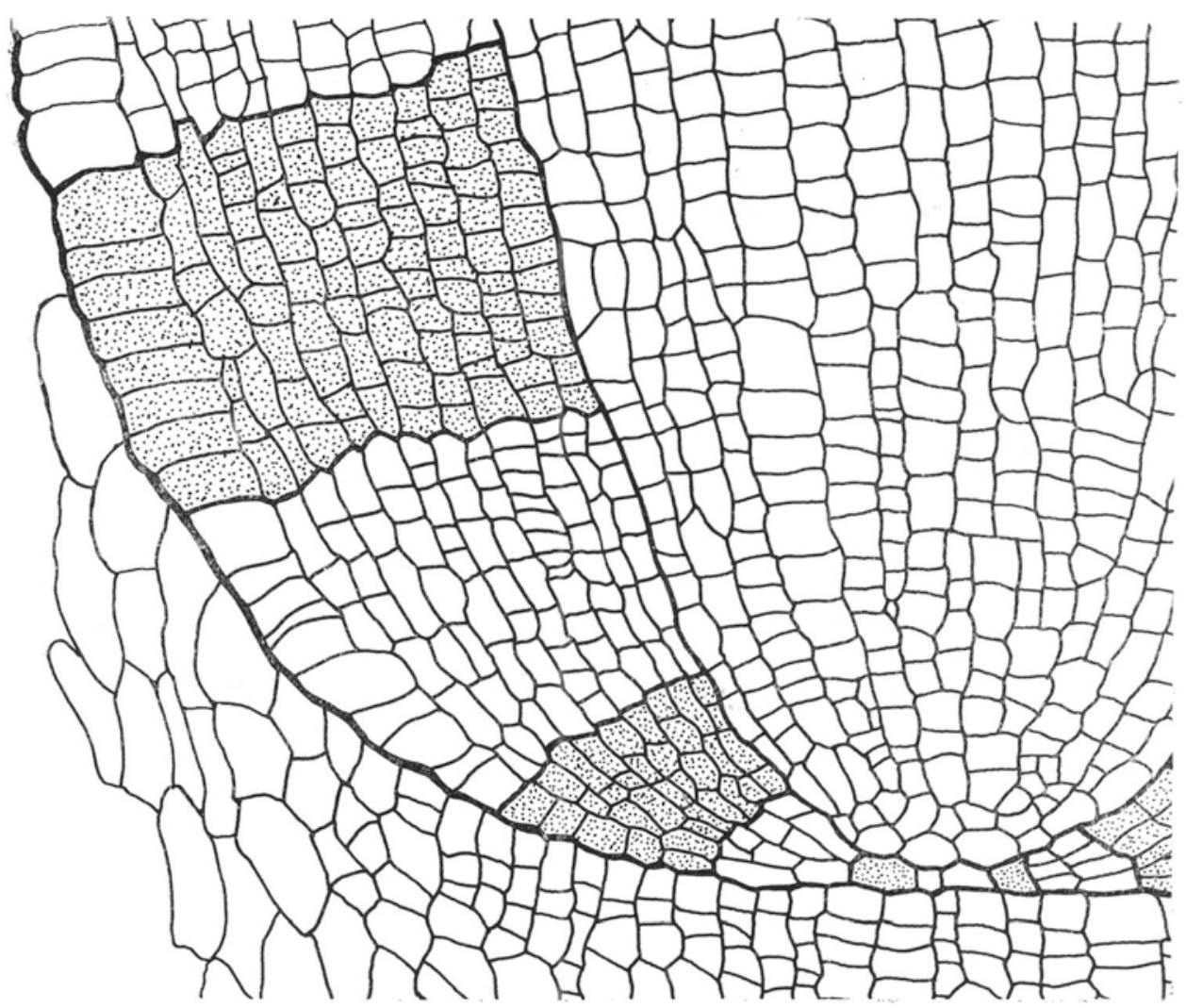

Fig. 1. Part of median section of the root apex of Zea mays showing the segmentation of the cortex-epidermis complex. Alternate segments are shaded.

is of the kind where it is possible to derive a quiescent centre on purely geometrical grounds in the same manner as it is in grasses (Clowes, 1961a). Concerning the question, discussed by $\mathrm{Kadej}$, of whether the central rows of cap cells (the columella) divide or not, it is not sufficient to examine only the median section in deciding whether longitudinal division of a cap initial has occurred. Nevertheless some longitudinal divisions do undoubtedly occur in the central cap initials especially when the apex is expanding laterally, but they are rare compared with the position in the peripheral cap initials. This is true of Zea as wells as of Cyperus and does not affect the geometrical proof of the existence of a quiescent centre for quiescence does not involve the complete absence of growth and mitosis (C lowes, 1961b, 1962; Clowes \& Hall, 1962).

However it is not the fact that some mitoses occur in the quiescent centre that explains the segmentation pattern. The pattern exists because, at an early stage in the formation of the root, there was a single 
cell (or a small group of cells) that did produce all the tissue now seen in the cortex-epidermis complex. The stage of which this is true is the very young root primordium in adventitious and lateral roots or the embryo in primary roots. At this early stage there is no quiescent centre; all the cells are meristematic (Clowes, 1961a). As the primordium grows, a quiescent centre is formed at the pole of the stele and cortex from cells that were previously meristematic. By this time the pattern of cells is already determined and the pattern seen in a mature root apex establishes the early behaviour of the primordium. In fact, the development of quiescence in the cells at the pole of the stele and cortex helps to preserve the original pattern as it was in the primordium.

There is therefore no conflict between the theories of the quiescent centre and the segmentation pattern in meristems so long as it is realized that the cell pattern reflects a primordial stage in the development of the root and not only its current behaviour.

Botany School, Oxford University,

England

\section{REFERENCES}

Clowes F. A. L., 1961a, Apical meristems, Blackwell Scientific Publications, Oxford.

Clowes F. A. L., 1961b, Duration of the mitotic cycle in a meristem, J. exp. Bot. $12: 283-293$.

Clowes F. A. L., 1962, Rates of mitosis in a partially synchronous meristem, New Phytol. 61:111-118.

Clowes F. A. L. \& Hall E. J., 1962, The quiescent centre in root meristems of Vicia faba and its behaviour after acute X-irradiation and chronic gamma irradiation, Radiation Botany 3:45-53.

$\mathrm{K}$ a d e j F., 1963, Interpretation of the pattern of the cell arrangement in the root apical meristem of Cyperus gracilis L. var alternifolius, Acta Soc. Bot. Polon. $32: 295-301$. 